\title{
Erratum: Influence of Dissipation on the Prefactor in the Expression of the Decay Rate of a Metastable State*
}

\author{
Yu. N. Ovchinnikov $\dagger$ \\ Istituto di Cibernetica del Consiglio Nazionale delle Ricerche, Naples, Italy
}

A. Barone

Istituto di Cibernetica del Consiglio Nazionale delle Ricerche, Naples, Italy, and Dipartimento di Fisica Nucleare, Struttura della Materia e Fisica Applicata, Università di Napoli, Naples, Italy

(Received January 25, 1988)

1. In Eq. (29), the coefficient preceding one of the $\zeta(3)$ functions should be replaced by 39 .

2. In Eq. (30), the term in the second set of braces

$$
-\frac{960}{\left(1+4|\alpha|^{2}\right)\left(1+|\alpha|^{2}\right)\left(9+4|\alpha|^{2}\right)}
$$

should be replaced by

$$
-\frac{300+240|\alpha|^{2}}{\left(1+4|\alpha|^{2}\right)\left(1+|\alpha|^{2}\right)\left(9+4|\alpha|^{2}\right)}
$$

This leads to the number $-2 \ln 2$ instead of the first three terms in the square bracket of Eq. (31). Therefore, in Eq. (33), $B / B_{0}=\exp \left(1.43 \eta / \eta_{2}\right)$, and in (34), $b=1.43$. This result differs just slightly from the numerical calculation by Grabert et al. (ref. 12) in our original paper). Also, in Eq. (33), $I=-0.182$.

We thank Prof. Peter S. Riseborough for his remarks, comments, and corrections.

\footnotetext{
*This paper appeared in J. Low Temp. Phys. 67, 323 (1987).

†Permanent address: Landau Institute for Theoretical Physics, Academy of Sciences of USSR, Moscow, USSR.
} 